\title{
El Contador Público en la era Digital
}

\section{The Certified Public Accountant in the Digital Age}

Miguel Ángel Chan Pereyra ${ }^{1}$. Germán Martínez Prats ${ }^{2}$, Sergio Alfonso Tosca Magaña ${ }^{3}$

${ }^{1}$ Universidad Juárez Autónoma de Tabasco (México)

chanpereyra@gmail.com

${ }^{2}$ Universidad Juárez Autónoma de Tabasco (México)

germanmtzprats@hotmail.com

https://orcid.org/0000-0001-6371-448X

${ }^{3}$ Sergio Alfonso Tosca Magaña

Universidad Juárez Autónoma de Tabasco (México)

sergio.alfonso9823@gmail.com

\section{DOI https://doi.org/10.46589/rdiasf.vi36.424}

Recibido 26 de junio 2021.

Aceptado 23 de septiembre 2021

Publicado 01 noviembre 2021

\section{Resumen:}

La tecnología es indispensable en nuestra vida cotidiana, así como en la vida laboral, un ejemplo de ello es la contabilidad, la cual es muy desgastante, pero con los avances tecnológicos ha sido de gran ayuda para los contadores, utilizando herramientas las cuales han simplificado esa labor que antes era tan laboriosa. En la presente investigación se describirá como se ha ido desarrollando las TICs y comoel contador se ha ido adaptando a las nuevas tecnologías para mejorar y desarrollar aún más sus capacidades en su área para así ofrecer a las empresas un servicio más eficiente y seguro.

Palabras clave: contabilidad, contador, herramienta, tecnologías. 


\begin{abstract}
Technology is indispensable in our daily lives, as well as in working life, an example of this is accounting, which is very exhausting, but with technological advances it has been of great help to accountants, using tools which have simplified that work that was previously so laborious. This research will describe how ICTs have been developed and how the accountant has been adapting to new technologies to improve and further develop its capabilities in its area to offer companies a more efficient and secure service.
\end{abstract}

Keywords: accounting, accountant, tool, technologies

\title{
Introducción
}

Los tics han evolucionado la manera de hacer las cosas, ya que se han desarrollo en diferentes áreas, en las cuales con ayudas de este tipo de herramientas se ha facilitado la manera de hacer las cosas esto conlleva a que las empresas se tengan que adaptar a estas nuevas herramientas tecnológicas las TIC’s, como nos describe Parrales (2017). Las tecnologías de información y comunicación incluyen todos los recursos informáticos que forman la base de los sistemas de información de una organización. Dentro de estos recursos, se incluyen productos y servicios tales como computadoras de escritorio, portátiles, dispositivos manejados, intranet cableada o inalámbrica, software de productividad empresarial como editor y hoja de cálculo, software empresarial, almacenamiento de datos y seguridad de red entre otros.

La demanda para disponer de estrategias de soluciones tecnológicas a los negocios para la administración y control de sus operaciones, propició a su vez el surgimiento de software y programas para el registro de la contabilidad y la administración de los recursos financieros, tanto para empresas grandes como para pequeños emprendedores, situación que favoreció la regulación en las disposiciones fiscales sobre el uso de la contabilidad 
electrónica al definir los parámetros y criterios tecnológicos para realizarla, así es como a partir de 2014 de forma legal en el Código Fiscal de la Federación (CFF), se estableció la obligación de registrar la información contable en medios electrónicos.

Los contadores han estado involucrados en el diseño de sistemas financieros durante décadas como consultores externos. En el pasado, los documentos de diseño estaban en el contexto de un sistema de gestión de registros manual. Hoy en día, los contadores quieren seguir brindando servicios similares, aunque en el contexto de las TIC.

Es por ello por lo que en la actualidad a profesión contable se ha tenido que adaptar para obtener el conocimiento en las TIC's.

\section{Método}

El diseño de la metodología tiene la tarea de determinar la organización de los procesos a desarrollar en una encuesta, para llevarla a cabo satisfactoriamente, determinar qué tipo de prueba se debe realizar y cómo se analizarán y verificarán los datos. (Sandoval \& Martínez, 2017) afirma que es: como una investigación descriptiva basada en documentos (materiales escritos u orales), con la finalidad de transformar las informaciones en datos cuantitativos (descripción objetiva, sistemática y cuantitativa del contenido manifiesto de una comunicación), y se compone de los pasos que se describen más adelante. Como es el caso de esta investigación en la cual se recabaron datos de diversos autores para poder conocer como el contador público se fue adaptando a las Tics para poder desarrollar su trabajo de una manera más fácil. De igual manera tendrá un enfoque exploratorio en cual buscaremos saber cómo las Tics han ido creciendo y así brindan las herramientas para facilitar ciertos procesos que eran manuales, pero ahora se hacen de manera digital. 


\section{Tecnologías de la información (TICS)}

Las TICS han sido una influencia en el desarrollo de las funciones del contador y han beneficiado de gran manera su labor dado que con el manejo de estás el contador a optimizado muchos procesos tales como de guardar o suministrar información a tiempo real. Como nos menciona Floréz (2012). Las TIC abren las fronteras y es allí se da una proliferación de empresas incrementando la competencia, aunque para muchas el acceso al mercado está limitado por su pensamiento; reducido a no participar en el mundo Web. El conocimiento de las tics y el uso de los programas son de vital importancia, específicamente cuando se refieren a manejo de información financiera.

De acuerdo Ramírez (2004). La tecnología de la información y de las comunicaciones son el conjunto de desarrollos tecnológicos relacionados con la captación, almacenamiento, manipulación, presentación y transmisión de datos que, basados en la utilización de procesamiento electrónico y de redes de difusión, se aplica a las actividades de cálculo, procesamiento, control, producción y comunicación de información.

Al pasar de los años se ha tenido un incremento significativo en el uso de las tecnologías de la información, esto se debe a que el uso de estas es más constante ya que nos brinda las herramientas para facilitarnos las tareas.

Suarez \& Sebastián (2017) informa que: "El uso masivo de las tecnologías de la información y comunicación como medios para generar, almacenar, transferir y procesar información se ha incrementado vertiginosamente en los últimos años, convirtiéndose en un elemento indispensable en gran parte de las organizaciones" (p.3). El diverso uso de dichas herramientas puede hacer que el desempeño de las personas no sea suficiente a la hora de entregar trabajos, tareas o proyectos.

La era digital tuvo inicio cuando se empezaron a crear las computadoras, cuando se crearon no tenían en cuentas el gran impacto que estas tendrían en el mundo y los alcance que esta misma tiene hasta ahora. Así mismo podemos ver como la contabilidad ha ido 
evolucionando y adaptándose al pasar de los años para poder brindar sus servicios a empresas. Los contadores se tienen que adaptar a los tics debido a que a través de estas se crean herramientas que ayudan a que el contador pueda realizar su trabajo de una manera más eficiente y segura, para que las empresas obtengan la información necesaria de una manera adecuada y rápida.

la digitalización sobre las formas de trabajar tiene que ver con la automatización de algunas tareas de funciones de soporte. De esta forma esta nueva tecnología favorece los procesos de automatización en la realización de tareas administrativas, favoreciendo el autoservicio por parte de los trabajadores en el proceso de la atención de cuestiones concretas: llenado de peticiones, formularios, solicitud de licencias, reembolso de gastos, reservaciones, etc. (Cedrola, 2017)

Con la ayuda de las TICs las organizaciones han logrado grandes beneficios, como es la optimización de sus recursos y la mejora de sus operaciones, conocer mejor las necesidades de los clientes para ofrecerles un mejor servicio, llegar a un mayor número de clientes y a nuevos mercados, y mejorar la comunicación entre sus empleados y con sus clientes y proveedores, logrando aumentar así su eficiencia. (Espinal, 2019).

Las tics generaron grandes cambios para el mundo sobre todo para las empresas que para poder seguir operando, y poder seguir siendo competentes en sus mercados se han tenido que adaptar a ellas, como se adapto el contador publico el cual se adapto a las herramientas creadas para facilitar su trabajo para realizarlo de una manera más sistematizada y haciéndolo mas rápido.

\section{Las TIC'S en el entorno laboral para el contador publico}

Las TIC con parte fundamental de la vida diaria, debido a que estas han ido evolucionando en diferentes aspectos, a los cuales nos hemos tenido que adaptar debido a que estas nos ayudan a poder hacer trabajos de una manera más eficiente y rápida. 
La era digital ha causado un gran impacto en la vida tanto cotidiana como laboral el autor, Jodar (2010). Destaca que las técnicas implantadas por esta nueva etapa digital constituyen un conjunto de tecnologías cuyas aplicaciones abren un amplio abanico de posibilidades a la comunicación humana. El carácter sinérgico de las nuevas tecnologías marcará los procesos productivos y comunicacionales de nuestra era a nivel mundial, denominada revolución Digital.

La innovación tecnológica en materia de TICs ha permitido la creación de nuevos entornos comunicativos y expresivos que abren la posibilidad de desarrollar nuevas experiencias formativas, expresivas y educativas, posibilitando la realización de diferentes actividades no imaginables hasta hace poco tiempo. (Ferro et. al., 2009).

Las TIC son fundamentales para un mayor crecimiento en el tema tecnológico debido a que muchas de las tareas que se hacían de manera manual ahora se hacen de manera digital, como lo es la banca en línea que, sin necesidad de ir al banco, todo lo puedas hacer desde un dispositivo con internet.

Las tecnologías de la información y la comunicación han transformado nuestra manera de trabajar y gestionar recursos. Las TIC son un elemento clave para hacer que nuestro trabajo sea más productivo: agilizando las comunicaciones, sustentando el trabajo en equipo, gestionando las existencias, realizando análisis financieros, y promocionando nuestros productos en el mercado. (Pita, 2018).

Las TIC tienen un impacto significativo en la productividad y eficiencia de las empresas, esto se debe a que muchos de los trabajos se han ido automatizando, otorgando esto una mayor facilidad para realizar ciertos trabajos.

Para Guatame \& Lagos (2020). el Contador Público debe desarrollar su capacidad innovadora para ofrecer mejores servicios, teniendo en cuenta que el producto de su trabajo es un elemento fundamental para que la empresa a su vez modernice sus productos, servicios y puedan contribuir con la eficiencia del mercado, 
Sin embargo, las TIC también pueden ser una fuente de distracción, debido que su uso es más frecuente, de igual manera en ciertas áreas dependemos mucho de las TIC en la cual sin ellas no podemos realizar ciertas tareas, de igual manera el uso de las TIC nos hace distanciarnos en ciertos aspectos de la realidad misma.

Escarraga (2019). Nos Menciona en su artículo en el cual nos habla sobre la evolución del contador que ha tenido con la tecnología que: En la actualidad podemos mirar como la tecnología con el pasar de los días ha tomado gran importancia en nuestra vida cotidiana debido que ha abarcado desde el campo académico hasta el laboral, generando cambios en nuestro quehacer y como profesionales de la contaduría pública también nos ha atrapado en este gran fenómeno, ya que el contador de antaño al momento de ejecutar varios proceso era laborioso porque a la hora de realizar un análisis o cuentas era mecánico, al instante de hacer cuentas o análisis de gran magnitud era algo largo y tedioso, con esto los procesos eran de días y conllevaba a tener errores pero con la llegada de la tecnología estos procesos poco a poco con el tiempos se fueron optimizando dichos métodos

La innovación es un cambio que alcanza a nuestro entorno diariamente, la tecnología es la principal causante de que esto suceda a través de nuevas invenciones, métodos y técnicas. "El comienzo de las prácticas contables se remonta desde tiempos muy antiguos, cuando el hombre se ve obligado a llevar registros y controles de sus propiedades porque su memoria no bastaba para guardar la información requerida" (Chávez, 2015). La mayoría de los sistemas informáticos especializados en contabilidad, tienen similitudes en su funcionamiento, crean pólizas de diario, ingreso, egreso, preparan información para ser enviada al portal del SAT, entre otras funciones, es de gran importancia que alumnos hayan usado el CONTPAQi y Aspel ya que, a la hora de laborar en su campo, tendrán conocimiento previo de cómo manejar un software contable.

El tratamiento de la información y la competencia digital implica ser una persona autónoma, eficaz, responsable, crítica y reflexiva al seleccionar, tratar y utilizar la información disponible, contrastándola cuando es necesario y respetar las normas de 
conducta acordadas socialmente para regular el uso de la información y sus fuentes en los distintos soportes. (MEC, 2006).

El papel fundamental que ha desempeñado la contabilidad es controlar y organizar las diferentes actividades de la empresa, con el fin de conocer la situación en que esta se encuentra, de una manera ágil, oportuna y útil, para llevar a cabo los propósitos que se quieren realizar y así cumplir con los objetivos. El sistema de información contable es una buena vía para que las empresas tengan una comunicación más adecuada tanto interno como externo, ya que por medio de la notificación que se estará dando en dichas empresas habrá una mejor organización y una buena toma de decisiones que los lleve a mejorar día a día y ser competitivos en el mercado. Como se puede ver en la siguiente tabla como fue la evolución de la tecnología en la contabilidad largo de los tiempos para facilitar la realización de esta:

Tabla 1

Evolución de la tecnología en la contabilidad.

\begin{tabular}{cccc}
\hline $\begin{array}{c}\text { Periodo } \\
\text { Histórico }\end{array}$ & $\begin{array}{c}\text { Necesidades } \\
\text { Informativas }\end{array}$ & $\begin{array}{c}\text { Posibilidades } \\
\text { Tecnológicas }\end{array}$ & $\begin{array}{c}\text { Respuesta de la } \\
\text { Contabilidad }\end{array}$ \\
\hline $\begin{array}{c}\text { Las grandes } \\
\text { civilizaciones }\end{array}$ & Conocer los ingresos y & Papiro, Escritura & Utilizar la partida \\
El inicio del & gastos & Cuneiforme & doble \\
Comercio & Registrar cada & Papel & Surge la partida \\
& movimiento & & doble Primeros \\
& & & Libros Contables \\
& Manejo de más & Los primeros & Se automatizan los \\
& información y con más & ordenadores, & sistemas contables \\
& rapidez & muchos usuarios & manuales \\
& & para un equipo & \\
& &
\end{tabular}




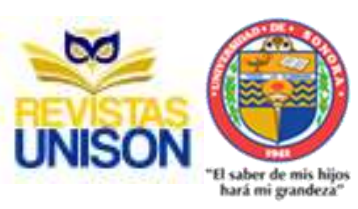

1981

Obtener información

financiera útil para la toma de decisiones

$$
\begin{gathered}
\text { Ordenador } \\
\text { personas, la } \\
\text { informática se } \\
\text { populariza }
\end{gathered}
$$

Sistema de información, contables integrados en base de datos informes

$\begin{array}{cc}\begin{array}{c}\text { Ordenadores en } \\ \text { red, internet, }\end{array} & \begin{array}{c}\text { Intercambio } \\ \text { electrónico de }\end{array} \\ \text { tecnologías de la } & \text { documentos, el } \\ \text { comunicación } & \text { papel es utilizado } \\ & \text { muy poco. }\end{array}$

Fuente: Guatame, B. y Lagos, L. (2017)

Las TIC's se han ido adaptando a las necesidades de las personas, tanto el ámbito cotidiano como el laboral, como lo es el caso de la contabilidad en la cual se han desarrollado diferentes tecnologías.

En la actualidad, la mayor parte de las transacciones comerciales pueden ser efectuadas en forma electrónica, sin contar con ningún tipo de documentación en papel, permitiendo a las organizaciones presentar información financiera en tiempo real, en línea. Debido al gran uso de las Tics las empresas se basan en tener el personal capaz de poder manejar y adaptarse a estas nuevas tecnologías para poder sacar el mejor provecho a todas las herramientas que estas nos brinda.

El conocimiento del grado de dominio de las competencias de TI requeridas por un profesional contable se convierte en un elemento fundamental para gestionar la información y el saber dentro de una organización. Las empresas actuales, requieren profesionales de la contabilidad capaces de potencializar los beneficios de los TICs con la finalidad de generar ventajas competitivas. A continuación, se describe la metodología empleada para conocer las principales competencias en TI requeridas por los contadores y su grado de dominio. (Martínez, et. al.,2017). 


\section{Conclusión}

La contabilidad a lo largo de los años ha ido creciendo de una manera eficiente y ha desarrollado herramientas para facilitar el trabajo del contador, y hacer que todo sea más eficiente y seguro, el contador se ha tenido que adaptar ala nuevas tecnologías para poder brindarle un mejor servicio a sus clientes, la contabilidad es uno pilar importante para las empresas ya que con ella se puede conocer los estados financieros para así tomar cualquier tipo de decisión. Con la ayuda de los tics la contabilidad se ha hecho de una manera eficiente al igual que organizada, los contadores públicos se han tenido que ir adaptando a estas tecnologías para poder brindar sus servicios ya que gracias a las tics las tareas del contador se facilitan demasiado, ya no se tiene que llevar un montón de papales para conocer la situación financiera, ahora todo puede ser guardado de manera digital y consultarlo desde cualquier lugar. El uso de las TICS le permite al contador, vivir siempre al tanto de las necesidades que demanda la sociedad, el mercado, la empresa y entre otros; por ello no solo hay que aprender sobre los tics, sino que hay que ir actualizándose continuamente, porque es de gran importancia las bases que los sistemas le brindan al contador. Las Tics brindan soportes para el desempeño laboral por medio del Software, como, EXCEL, ACCES, y Paquetes Contables, que permiten simplificación y rapidez en el trabajo. En conclusión, en esta investigación se describió como ha sido la adaptación del contador a la era digital, debido a que esta misma esta siempre yen crecimiento y con esto conlleva a tener mejores tecnologías que buscan el facilitar y brindar ayuda al trabajo de ciertas personas. Los tics se volvieron una parte importante de la vida de las personas, así como de los contadores debido a las grandes facilidades que te lleva el hacer una tarea por medio de la tecnología. El contador tendrá que ir adaptándose a los nuevos cambios tecnológicos ya que estos le proporcionarán las herramientas necesarias para que pueda realizar su trabajo de una manera eficiente y segura. 


\section{LITERATURA CITADA}

1. Chávez, J. (2015). Contabilidad electrónica, un paso fuerte a la modernización contable. Ciencias Empresariales, 25, 70-86.

2. Guatame, B. y Lagos, L. (2017) Retos del contador público frente a los cambios en la era digital en Colombia. En: Criterios, Revista de Estudiantes Facultad de Ciencias Económicas. UMNG. Vol.VII N${ }^{\circ}$.

3. Izaguirre, J. G. (2014). PRINCIPIOS DE CONTABILIDAD GENERALMENTE ACEPTADOS Vigencia y Aplicación. Quipukamayoc, 7(13), 121-134.

4. Kimura, E. B. S. El sistema contable en la nube-Diagnóstico actual y desafíos con la unificación de códigos. 73-Cra. Elsa Beatriz Suarez Kimura-Universidad de Buenos Aires y Dr. Diego Sebastián Escobar-Universidad del Salvador.

5. Flórez Castañeda M. A. (2012). ERA DIGITAL, LA NUEVA REALIDAD DEL CONTADOR PÚBLICO. Adversia, (1). Recuperado a partir de https://revistas.udea.edu.co/index.php/adversia/article/view/11299

6. MEC], i. d. (2006). Real Decreto 1513/2006, de 7 de diciembre, por el que se establecen las enseñanzas mínimas de la Educación primaria. Obtenido de https://www.boe.es/boe/dias/2007/01/05/pdfs/A00677-00773.pdf

7. Moreno, J. M., Obregón Angulo, M. D., \& García Martínez, C. (2016). Las nuevas capacidades tecnologías de los contadores públicos en México. Global de Negocios, 4(3), 12 .

8. Sandoval, K. A., \& Martínez, F. (2017). Tendencias en el diseño metodológico de investigación sobre la evaluación de competencias en la educación superior. REIRE, 13. 
9. Escarraga Beleño, J. A. (2019). Evolución del contador público frente a la era digital [Trabajo de pregrado, Universidad Cooperativa de Colombia]. Repositorio Institucional UCC. Disponible en: http://hdl.handle.net/20.500.12494/33177

10. RODRIGUEZ DE RAMIREZ, M. D. C. (2004). LA CONTABILIDAD Y EL IMPACTO DE LAS TECNOLOGÍAS DE LA INFORMACIÓN Y LAS COMUNICACIONES. Contabilidad Y Auditoría, (19), 22 Págs. Disponible en: https://ojs.econ.uba.ar/index.php/Contyaudit/article/view/144

11. Ferro Soto, C., Martínez Senra, A. I., \& Otero Neira, M. C. (2009). Ventajas del uso de las TICs en el proceso de enseñanza-aprendizaje desde la óptica de los docentes universitarios españoles. Edutec: Revista electrónica de tecnología educativa. Disponible en: http://hdl.handle.net/11162/6022

12. Pita, G. E. C. (2018). Las TICs en las empresas: evolución de la tecnología y cambio estructural en las organizaciones. Dominio de las Ciencias, 4(1), 499-510. Disponible en: https://dialnet.unirioja.es/servlet/articulo?codigo=6313252

13. Guatame Rodríguez, B., \& Lagos Botia, L. G. (2020). Retos del contador público frente a los cambios en la era digital en Colombia. Criterios Revista Estudiantes Facultad De Ciencias Económicas, 7(1), 49-57. Disponible en: https://revistas.unimilitar.edu.co/index.php/CREFCE/article/view/4821

14. Parrales-Villacreses, J. (2017). Gerencia Estratégica y Tecnología de la Comunicación e Información -TICs. Dominio de las Ciencias, 3(3 mon), 218-236. Disponible

en: https://www.dominiodelasciencias.com/ojs/index.php/es/article/view/637

15. Cedrola Spremolla, G. (2017). El trabajo en la era digital. Revista De Derecho, $16(31)$, 103-123.

Disponible

en: http://revistas.um.edu.uy/index.php/revistaderecho/article/view/453 
16. Jódar Marín, J. A. (2010). LA ERA DIGITAL: NUEVOS MEDIOS, NUEVOS USUARIOS Y NUEVOS PROFESIONALES. Razón y Palabra, (71), Disponible en: https://www.redalyc.org/articulo.oa?id=199514914045

17. Martínez, G. M. F., Montemayor, T. D. J. E., \& Torres, E. G. C. (2017). Gestión estratégica y efectividad en las tecnologías de información: reto para la profesión contable.Enl@ ce: Revista Venezolana de Información, Tecnología y Conocimiento, 14(1), 9-27. Disponible en: https://dialnet.unirioja.es/servlet/articulo?codigo $=6068215$

18. Espinal, A. . (2019). El papel de las TIC en las empresas. Revista TecnológicaEducativa Docentes 2.0, 1(1), 13-14. Disponible en:

https://ojs.docentes20.com/index.php/revista-docentes20/article/view/38

Cómo citar:

Martínez Prats, G., Chan Pereyra, M. ., \& Tosca Magaña, S. . (2021). El Contador Público en la era Digital. Revista De Investigación Académica Sin Frontera: División De Ciencias Económicas Y Sociales, (36). https://doi.org/10.46589/rdiasf.vi36.424
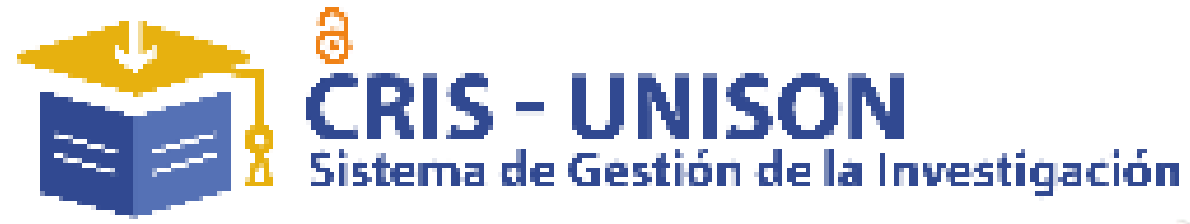

- Dialnet

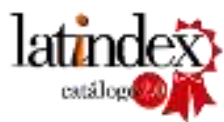

6f IIIFACTOR

REDIB

*iditeractor

2LatinREV Neliti - Indonesia's Research Repository
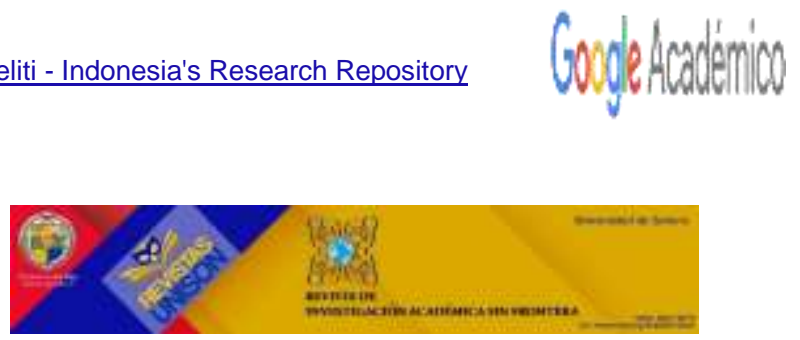